\title{
Do external stimuli, applied to train cultured cortical networks, disturb the balance between activity and connectivity?
}

\author{
Joost le Feber, Jan Stegenga, and Wim Rutten, Member, IEEE
}

\begin{abstract}
Learning, or more generally, plasticity may be studied using cultured neuronal networks on multi electrode arrays. Many protocols have been proposed to change connectivity in such networks. So far, only one of these protocols, proposed by Shahaf and Marom, aimed to change the input-output relationship of a selected connection in the network. Although the results were quite promising, the experiments appeared difficult to repeat and the protocol did not serve as a basis for wider investigation yet. Here, we repeated their protocol, and compared our 'learning curves' to the original results. Although in some experiments the protocol did not seem to work, we found that on average, the protocol showed a significant learning effect indeed. We frequently found learning curves that initially declined as in the original results, but then increased again before finally settling at a low level.
\end{abstract}

\section{INTRODUCTION}

A CTIVITY patterns in neuronal networks depend on the connections in the network. On the other hand, activity patterns may also change network connectivity. It appears that mature networks develop a balance between activity and connectivity. External (electrical) stimuli, as commonly used in plasticity studies, may disturb the equilibrium, forcing the network to find a new balance.

Several studies investigated plasticity in cultured neuronal networks, but only the protocol by Marom and Shahaf [1] aimed to train a culture to produce a predefined response upon stimulation. Although their results seemed quite successful, to our knowledge, they were never reproduced elsewhere and they did not serve as a basis for wider exploration yet. In this study we tried to reproduce their results. To investigate the effects of a potentially disturbed activity-connectivity balance, we extended the analysis interval from $\sim 12$ test cycles to $\sim 40$ and we looked at longer term effects of the protocol

\section{METHODS}

\section{A. Cell cultures}

Cortical cells were obtained from newborn Wistar rats. After trypsin treatment cells were dissociated by

Manuscript received April 16, 2008.

All Authors are with the institute of Biomedical Technology, Department of Electrical Engineering, Mathematics and Computer science, Biomedical Signals and Systems Group, University of Twente, PO Box 217, 7500 AE, Enschede, The Netherlands (corresponding author phone: +31.53.489.2790, e-mail: j.lefeber@ utwente.nl). trituration. About 400,000 dissociated neurons (400 $\mu \mathrm{l}$ suspension) were plated on a MEA precoated with poly ethylene imide. This procedure resulted in a cell density of approximately 5000 cells per $\mathrm{mm}^{2}$.

Neurons were cultured in a circular chamber (inner diameter: $20 \mathrm{~mm}$ ) glued on top of a multi electrode array (MEA) with 60 electrodes. The culture chamber was filled with $700 \mu \mathrm{l} \mathrm{R} 12$ medium [2]. MEAs were stored in an incubator, under standard conditions of $37^{\circ} \mathrm{C}, 100 \%$ humidity, and $5 \% \mathrm{CO}_{2}$ in air. For recording, we firmly sealed the culture chambers with a watertight but $\mathrm{CO}_{2}$ permeable foil, and placed the cultures in a measurement setup outside the incubator. During recording we maintained the $\mathrm{CO}_{2}$ level around 5\%. For details about the recording setup see [3].All recordings were started after an accommodation period of at least 20 minutes.

After the measurements the cultures were returned to the incubator.

We used 8 different cultures for 10 experiments, which were done $26 \pm 14$ days after plating of the dissociated cells.

\section{B. Select electrodes}

In all experiments, first, all electrodes were stimulated at various amplitudes to select a stimulation electrode that frequently induced a network burst and (following the original training protocol) an evaluation electrode that responded to these stimuli at a ratio of $\sim 0.1$. We plotted curves of the number of action potentials at each electrode, as a function of the latency to the stimulus. Figure 1A shows an example of the probability to record an action potential at the evaluation electrode as a function of the latency after the stimulus. (the 'responsiveness' of a selected electrode). Response curves of evaluation electrodes usually had a peaked shape, similar to that in Fig.1A. The first peak around zero latency was probably caused by some residual stimulus artifact, or by non-synaptically transmitted direct responses, through retrograde stimulation of axons. We focused on the second peak, around $20 \mathrm{~ms}$ in the example in Fig. 1

We selected evaluation electrodes that had a response ratio of $\sim 0.1$ in a time window around the maximum of the second peak. Time windows had a width of 20-50 ms and were adjusted to obtain this response ratio. In the example of Figure 1 the evaluation time window was set to $10-40 \mathrm{~ms}$.

\section{Training protocol}

We stimulated the selected electrode until the evaluation electrode showed at least 2 responses to the 
last 10 stimuli or until the maximum stimulation time of $10 \mathrm{~min}$ was reached. When the threshold (or the maximum stimulation time) was reached, stimulation stopped automatically, followed by 5 minutes of no stimulation. We repeated this cycle until the network wide response to the stimuli dropped below threshold in three consecutive cycles. This threshold was set to $80 \%$ of the average response to the first 5 stimuli.

We plotted the number of applied stimuli against the cycle number (a learning curve), and interpreted a decreasing number of stimuli as a learning effect.
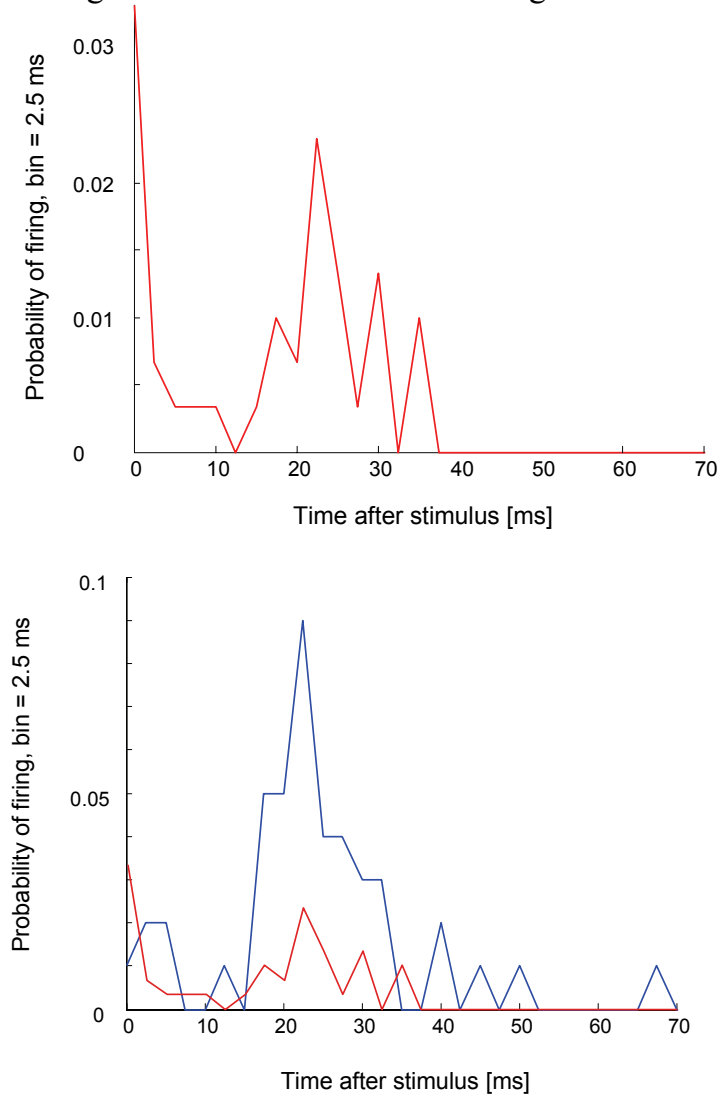

Figure 1. A shows the 'responsiveness' of a selected evaluation electrode before the training protocol. The time interval to detect responses was set at $10-40 \mathrm{~ms}$, such that the summed probability before training was about 0.1 . B shows the responses to the first 10 stimuli of the training experiment (red) and the responses to the last 10 stimuli (blue)

\section{Connectivity analysis}

Before and after the training protocol we recorded one hour of spontaneous activity to analyze network connectivity. For all possible pairs of electrodes $(60 \times 59)$ we calculated conditional firing probabilities (CFP's) as the probability to record an action potential at electrode $j$ at $\mathrm{t}=\tau$, given that one was recorded at electrode $\mathrm{i}$ at $\mathrm{t}=0$. If a CFP curve was not flat, the two neurons were functionally connected. An example is shown in Figure 2. This functional connection may be described by two parameters: strength and latency [4]. These parameters may be used to follow the development of a functional connection in time [5].

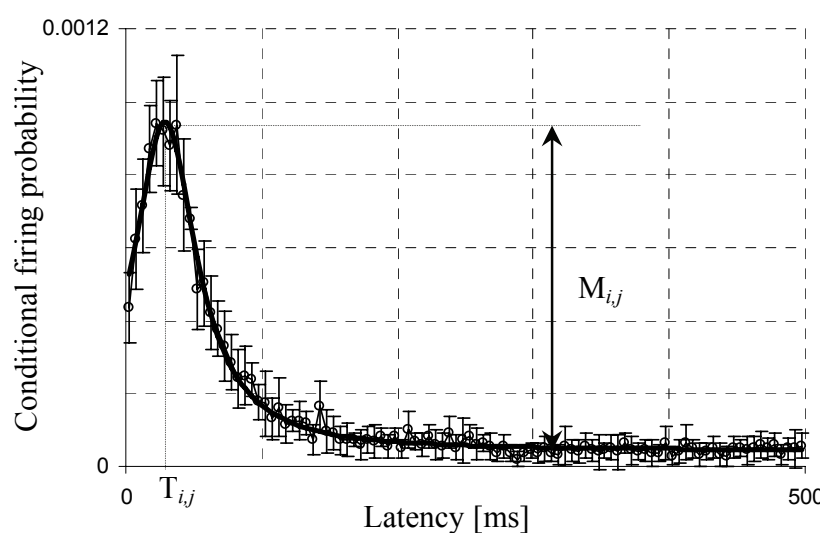

Figure 2. Example of estimated conditional firing probability $(\circ$, mean $\pm \mathrm{SD}$ of 5 consecutive bins of $0.5 \mathrm{~ms}$ each). Solid line represents fitted equation, used to obtain values for strength $\left(\mathrm{M}_{i, j}\right)$ and latency $\left(\mathrm{T}_{i, j}\right)$ of the functional connection between a pair of electrodes $(i, j)$.

\section{RESULTS}

We applied the training protocol in 10 experiments. Figure 1B shows an example of the responses to the first 10 stimuli (red line) and to the last 10 stimuli (blue line) of the training protocol in a successful experiment.
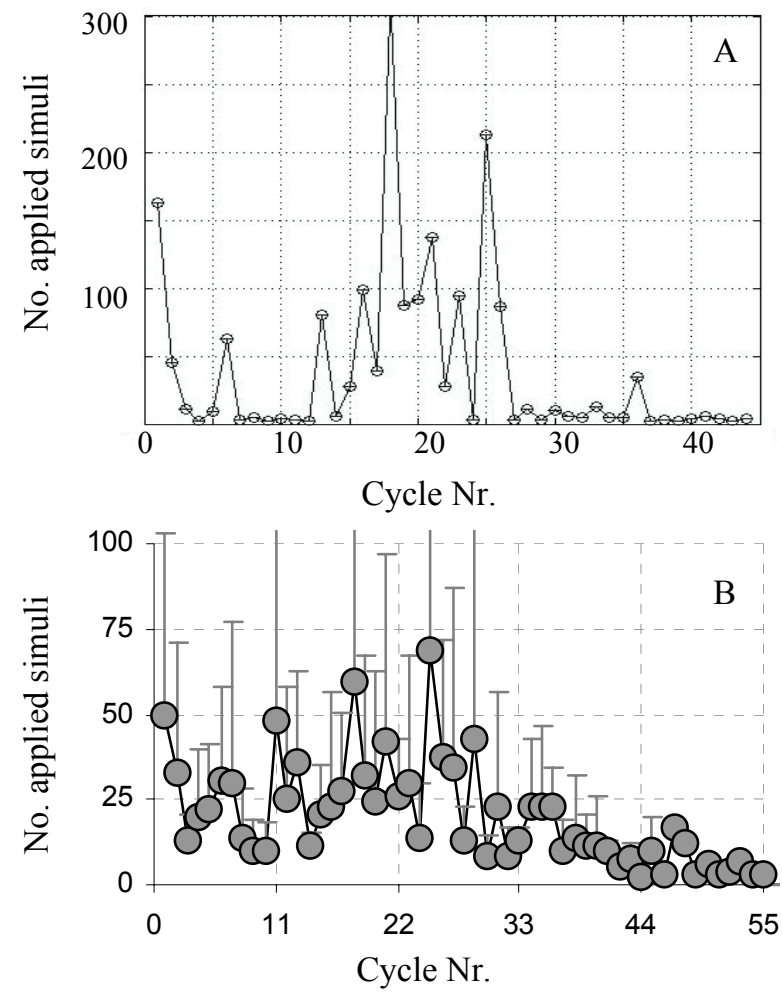

Figure 3. Learning curves resulting from training protocol. A shows a typical example of an individual learning curve, as observed in 5 of 10 experiments. B depicts average learning curve $(\bullet$, mean \pm SD) of all 10 experiments. No. applied stimuli decreases significantly with trial Nr. (Kendall's tau: Correlation coefficient: $-0.33 ; \mathrm{P}<0.01$ ). 
In $50 \%$ of all experiments we found a learning curve as in the example of Figure 3A, which may be characterized by an initial decline (continuing for $14 \pm 8$ cycles, mean \pm $\mathrm{SD}$ ), followed by a rise, roughly centered around trial $\mathrm{Nr}$

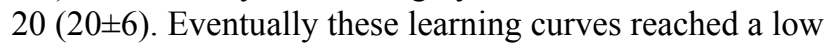
level as in Figure 3A. The width of the increase around trial Nr. 20 varied and averaged $13 \pm 7$ cycles.

In another $30 \%$ a stable low level was reached immediately, whereas $20 \%$ showed wild fluctuations without a clear trend. The average learning curve of all cultures, including the $20 \%$ without a clear trend, is shown in Fig. 3B. Strikingly, the first 10 trials yielded results very similar to the original results published by Shahaf and Marom [1]. However, after the $10^{\text {th }}$ cycle the average number of stimuli increased again. This phenomenon was seen in 5 individual experiments. Because the centre of the rise differed between experiments, on average the effect was somewhat blurred out, resulting in a lower and wider second peak in the averaged curve, as well as higher standard deviations (Fig

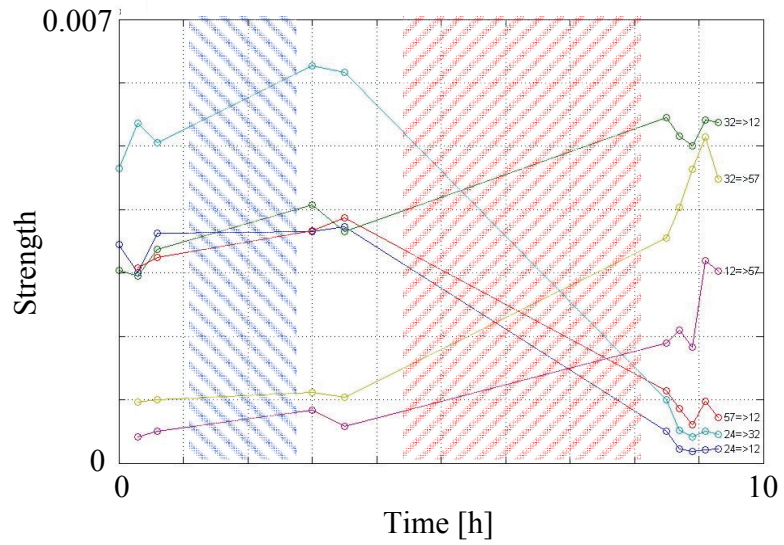

Figure 4. Strengths of persisting connections during one of our experiments. The experiment consisted of 5 phases. White areas: spontaneous activity recordings. Blue bar: random stimulation to select electrodes (see section II.B). Red bar: training protocol (see section II.C). The graphs illustrate that the strength of most individual connections was affected by the protocol; either strengthened or weakened. In total, the strength of $64 \%$ of all persisting connections was significantly changed. The figure also suggests that global parameters like mean strength may not be affected by the protocol.

3B).

Finally, we used spontaneous activity recorded before and after the training protocol to investigate connectivity changes in the network. Occasionally new functional connections appeared or existing ones disappeared during the training protocol, but on average this number was small, compared to the number of persisting functional connections. The strength of $64 \%$ of all persisting connections was significantly affected by the protocol, either up or down. Figure 4 shows an example with only very few persisting functional connections. Usually there were many more persisting connections and the depicted example is not representative in this respect. However, the selected example clearly shows that the strength of functional connections changed substantially, both up and down Although the average strength did not change, the mean absolute change was $52 \pm 26 \%$, clearly exceeding the spontaneous fluctuation during a comparable time span ( $\sim 30 \%$ standard deviation, see [4]).

\section{Discussion}

Our results show that the applied training protocol was effective in $50 \%$ of all cultures. It may be argued that the protocol was also effective in the $30 \%$ of experiments that immediately reached a stable low level. In these experiments, however, the results were more difficult to interpret because the 'learning curves' did not show any improvement. In $20 \%$ the training protocol did not reduce the number of input stimuli needed to reach the desired output. However, analysis of spontaneous activity before and after the protocol showed that in all experiments, including these unsuccessful ones, the strength of a major part of all functional connections was changed. This means that, even though the protocol sometimes could not induce a chosen modification, it always did affect network connectivity.

How is it possible that the success of the protocol varies so much? One possible explanation is the balance that cultured networks may develop between activity and connectivity. Because activity patterns arise from certain connectivity, and activity, in turn, influences connectivity, the finding that networks develop stable activity patterns $[4,6]$ may be interpreted as an established balance between activity and connectivity.

If external stimulation pushes the network out of balance, it may develop towards a new equilibrium which may or may not include the selected connection.

Thus the choice of the connection to be trained may determine the success of the protocol, but one cannot predict whether or not a selected connection will lead to a success. On average, however, we did find a significantly 'improved' response to electrical stimuli after the training protocol.

Another interesting phenomenon is the rise around trial 20 , after an initial decrease. Because it was observed in $50 \%$ of the experiments, it seems unlikely that this occurred purely coincidentally. This is emphasized even more by the fact that in a later paper Marom et al observed a similar increase around stimulation cycle 18 in an averaged leaning curve of 16 experiments [7]. Unfortunately, the scale of the graph (Fig. 10 in their paper) was adapted to include another curve, which masked the effects, and it is not further addressed in their paper.

Is it possible that this second peak in our learning curves is caused by a reorganization of the whole network? Is it possible that initially an individual connection can be changed, while the network is in an unbalanced state, and that internal forces will then drive the network into a new balance that may or may not 
contain the alteration of the selected connection. Could it be that in the $30 \%$ group, the new balance did contain the chosen alteration of the selected connection, whereas in the $50 \%$ group there was a conflict between intended change and newly found balance? In this latter situation, it would be fully comprehensible that it took so much longer before a stable low level was reached. It is even possible that the learning curves of the $20 \%$ unsuccessful experiments would eventually have reached a stable low level if we could have continued measurements long enough.

What we can conclude is that the learning protocol did affect network connectivity in all experiments, that on average we found a descending learning curve. However, individual curves usually did not descend very smoothly, suggesting a more complicated mechanism than just strengthening of a synaptic pathway.

\section{ACKNOWLEDGMENT}

The authors would like to thank prof.dr. E. Marani and R. Wiertz for their work on the preparation and maintenance of cultures.

\section{REFERENCES}

[1] G. Shahaf and S. Marom, "Learning in networks of cortical neurons.," J. Neurosci., vol. 21, pp. 87828788, 2001.

[2] H. J. Romijn, F. van Huizen, and P. S. Wolters, "Towards an improved serum-free, chemically defined medium for long-term culturing of cerebral cortex tissue," Neurosci. Biobehav. Rev., vol. 8, pp. 301-334, 1984.

[3] J. Stegenga, J. le Feber, E. Marani, and W. L. C. Rutten, "Analysis of cultured neuronal networks using intra-burst firing characteristics," IEEE Trans. Biomed. Eng.., vol. 55, pp. 1382-1390, 2007.

[4] J. le Feber, W. L. C. Rutten, J. Stegenga, P. S. Wolters, G. J. Ramakers, and J. Van Pelt, "Conditional firing probabilities in cultured neuronal networks: a stable underlying structure in widely varying spontaneous activity patterns," J. Neural Eng., vol. 4, pp. 54-67, 2007.

[1] [5]J. le Feber, J. Van Pelt, and W. L. C. Rutten, "Latency dependent development of related firing patterns in cultured neuronal networks," presented at IEEE-EMBC, Lyon France, 2007.

[6] J. van Pelt, P. S. Wolters, M. A. Corner, W. L. C. Rutten, and G. J. Ramakers, "Long-term characterization of firing dynamics of spontaneous bursts in cultured neural networks.," IEEE Trans. Biomed. Eng., vol. 51, pp. 2051-2062, 2004.

[7] S. Marom and G. Shahaf, "Development, learning and memory in large random networks of cortical neurons: lessons beyond anatomy," Q.Rev. Biophys, vol. 35, pp. 63-87, 2002. 